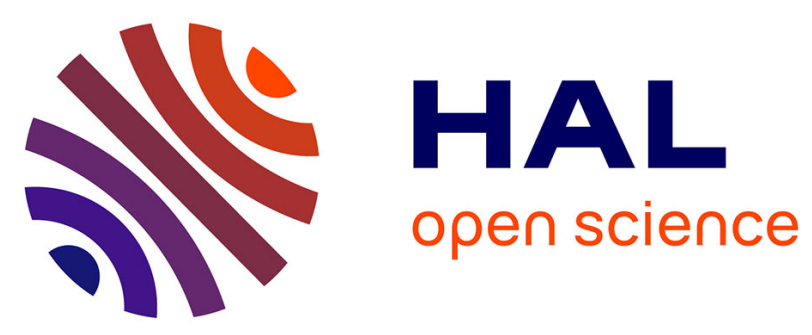

\title{
Morphogenesis mechanisms in the hydrothermal growth of lead-free BCZT nanostructured multipods
}

Zouhair Hanani, Ablouh El-Houssaine, Soukaina Merselmiz, Jaafar Ghanbaja, M'barek Amjoud, Daoud Mezzane, Abdelhadi Alimoussa, Mohammed Lahcini, Matjaž Spreitzer, Damjan Vengust, et al.

\section{To cite this version:}

Zouhair Hanani, Ablouh El-Houssaine, Soukaina Merselmiz, Jaafar Ghanbaja, M'barek Amjoud, et al.. Morphogenesis mechanisms in the hydrothermal growth of lead-free BCZT nanostructured multipods. CrystEngComm, 2021, 23 (30), pp.5249-5256. 10.1039/D1CE00591J . hal-03342924

\section{HAL Id: hal-03342924 https://hal.science/hal-03342924}

Submitted on 14 Sep 2021

HAL is a multi-disciplinary open access archive for the deposit and dissemination of scientific research documents, whether they are published or not. The documents may come from teaching and research institutions in France or abroad, or from public or private research centers.
L'archive ouverte pluridisciplinaire HAL, est destinée au dépôt et à la diffusion de documents scientifiques de niveau recherche, publiés ou non, émanant des établissements d'enseignement et de recherche français ou étrangers, des laboratoires publics ou privés. 


\title{
Morphogenesis mechanisms in the hydrothermal synthesis of lead-free BCZT nanostructured multipods ${ }^{\dagger}$
}

Received 00th January 20xx, Accepted 00th January 20xx DOI: $10.1039 / \times 0 \times x 00000 x$

\begin{abstract}
Zouhair Hanani, ${ }^{a, b, c}$ El-houssaine Ablouh, ${ }^{d}$ Soukaina Merselmiz, ${ }^{a}$ Jaafar Ghanbaja, ${ }^{e}$ M'barek Amjoud, ${ }^{a}$ Daoud Mezzane, ${ }^{a, f}$ Abdelhadi Alimoussa, ${ }^{a}$ Mohammed Lahcini, ${ }^{a}$ Matjaž Spreitzer, ${ }^{c}$ Damjan Vengust, ${ }^{\mathrm{C}}$ Mimoun El Marssi, ${ }^{\mathrm{f}}$ Igor A. Luk'yanchuk, ${ }^{\mathrm{f}, \mathrm{g}}$ Zdravko Kutnjak, ${ }^{\mathrm{c}}$ and Mohamed Gouné $^{\mathrm{b}, *}$

Due to growing environmental concerns on the toxicity of lead-based piezoelectrics, the replacement of $\mathrm{Pb}$-based materials by the homologs with comparable piezoelectric properties but without lead is an emergent task. Since 2009, $\mathrm{Ba}_{0.85} \mathrm{Ca}_{0.15} \mathrm{Zr}_{0.1} \mathrm{Ti}_{0.9} \mathrm{O}_{3}$ (BCZT) material has aroused considerable attention for such replacement due to its excellent dielectric, ferroelectric and piezoelectric properties. Here, templated-growth of BCZT nanostructured multipods with hydrogen zirconate titanate nanowires (HZTO-NWs) was investigated under hydrothermal conditions. The effect of the precursor's concentrations and the hydrothermal reaction time on the morphological formation of BCZT nanostructures were investigated. Besides, the composition, structure and phases analysis studies were carried out, and the growth mechanism of BCZT multipods was proposed. It was found that the precursor's concentrations and dwell time in hydrothermal reaction play a critical role in the formation of BCZT multipods, and the desirable BCZT phase was obtained in samples using low barium and calcium concentrations and at low reaction time. This research has a general validity and can be extended to design more complex perovskite oxides.
\end{abstract}

\section{Introduction}

Because of their enhanced dielectric, ferroelectric and piezoelectric properties, $\mathrm{ATiO}_{3}(\mathrm{~A}=\mathrm{Ca}, \mathrm{Sr}$, and $\mathrm{Ba})$ oxides with perovskite structure are the most intensively investigated materials for applications in ceramic capacitors, sensors, transducers, and nonvolatile memories. $^{1-3}$ These physical properties are strongly dependent on the compositions, features and morphologies of $\mathrm{ATiO}_{3}$ perovskite materials which have been largely elaborated by solid-state, $^{4,5}$ sol-gel, $^{6,7}$ co-precipitation, ${ }^{8}$ and hydrothermal methods. $^{9-11}$ In particular, this later offers various advantages such as creating crystalline phases which are not stable at the melting point, the ability to synthesise large crystals of high-quality, and the possibility to design nanostructured materials with specific morphologies. ${ }^{10,12}$ Besides, the nature of the precursors used in hydrothermal synthesis often plays a key role in determining the shape and size of the resulting nanomaterials. ${ }^{10,13}$ The nucleation and growth of $\mathrm{ATiO}_{3}$ from the organometallic, ionic or oxide B-site precursors in the presence of A-site carbonates or salts are often

\footnotetext{
a. IMED-Lab, Cadi Ayyad University, Marrakesh, 40000, Morocco

b. ICMCB, University of Bordeaux, Pessac, 33600, France

"E-mail:mm.goune@gmail.com

c. Jozef Stefan Institute, Ljubljana, 1000, Slovenia

d. MSN, Mohammed VI Polytechnic University, Ben Guerir, 43150, Morocco

e. IJL UMR 7198 CNRS, University of Lorraine, 54000, Nancy, France

f. LPMC, University of Picardy Jules Verne, Amiens, 80039, France

g. Department of Building Materials, Kyiv National University of Construction and

Architecture, Kyiv, Ukraine

tElectronic supplementary information (ESI) available.
}

dictated by the cubic crystal structure, which results in the formation of $\mathrm{ATiO}_{3}$ nanocrystals with isometric morphologies (spherical or cubic shapes). ${ }^{10}$ It was reported that the nature of the solid precursor can highly influence the crystallization pathway of the perovskite materials. ${ }^{10,14}$ The template growth approach is beneficial to tune the shape of inorganic nanoparticles, because the template's surface provides sites for further growth by depleting the precursor monomer in bulk solution. ${ }^{15}$ Kalyani et al. investigated the effect of four types of solid titanium as reactive templates precursors (anatase, rutile, sodium titanate, and hydrogen titanate) of nanowire morphology, on the hydrothermal crystallization of $\mathrm{SrTiO}_{3}{ }^{14}$ Various products i.e., mesocrystals, polycrystalline, isolated particles or with dendritic morphology could be found. Besides, the use of layered alkali-metal or layered protonic titanates precursors has been found to have a substantial impact on both the phase formation and crystal growth of perovskite materials. ${ }^{16}$ The crystallographic structure of layered alkali-metal or layered protonic titanates precursors corresponds to anatase-like layers consisting of corner-shared $\mathrm{TiO}_{6}$ octahedra separated by interlayers containing alkaline and variable quantities of $\mathrm{H}_{2} \mathrm{O} .{ }^{17}$ Owing to the layered structure and weaker interactions along the stacking direction, the alkaline ions can be easily exchanged with ions of similar size, such as alkaline-earth cations. ${ }^{18}$ In a topochemical transformation, both the morphology and the crystallographic order of the template are preserved, thus enabling a high level of morphological control. ${ }^{11}$ In some cases, compounds with a wire-like morphology are generated as intermediate products during the hydrothermal treatment and act as effective 
reactive templates for the synthesis of $1 \mathrm{D}$-pervskite materials. ${ }^{19} \mathrm{Zhu}$ et al. reported the synthesis of $\mathrm{BaTiO}_{3}$ nanotubes via a simple wet chemical route at low temperature $\left(50{ }^{\circ} \mathrm{C}\right)$ using $\mathrm{H}_{2} \mathrm{Ti}_{3} \mathrm{O}_{7}$ nanotubes as a template. ${ }^{20}$ Moreover, Yang et al. prepared a single crystalline, uniform size distribution and ultra-high aspect ratio $\mathrm{BaTiO}_{3}$ nanowires via a simple one-step hydrothermal treatment. ${ }^{21}$ It was found that the synergistic effects including both Ostwald ripening and cation exchange reaction are responsible for the growth of the ultrathin $\mathrm{BaTiO}_{3}$ nanowires. Nevertheless, in numerous cases of templated growth perovskite, a topochemical transformation of the template to the perovskite materials is not observed due to the domination of the heterogeneous nucleation of the perovskite on the surface of the template and dissolution-precipitation processes rather than ionic exchange during the hydrothermal reaction. ${ }^{9,11,12,14,22}$ Accordingly, polycrystalline wires with a rough surface, dendritic crystals or even loose nanoparticles are observed. ${ }^{11,14}$ A controlled hydrothermal synthesis of $\mathrm{BaTiO}_{3}$ nanostructures (nanowires, nanosheets, nanocubes, starfish-like, and sword-like) using $\mathrm{Na}_{2} \mathrm{Ti}_{3} \mathrm{O}_{7}$ nanotubes and nanowires as synthetic precursors was reported by Bao et al. ${ }^{10}$ However, the majority of the reported works basing on templated hydrothermal growth using layered alkali-metal or layered protonic titanates precursors are focusing on the preparation of simple perovskite oxide like $\mathrm{BaTiO}_{3}, \mathrm{CaTiO}_{3}$ and $\mathrm{SrTiO}_{3}$. Though, the most fascinating dielectric, ferroelectric and piezoelectric properties are found in complex perovskite oxides.

In 2009, Liu and Ren proposed $\mathrm{Ba}_{0.85} \mathrm{Ca}_{0.15} \mathrm{Zr}_{0.1} \mathrm{Ti}_{0.9} \mathrm{O}_{3}$ (BCZT) ceramic with remarkable electrical properties; as a milestone discovery in the field of ferroelectrics, ${ }^{23}$ which make it a promising material for energy storage and energy harvesting applications. ${ }^{24-29}$ The majority of published works is based on high temperature solidstate reaction to make pure and crystalline BCZT powders, ${ }^{27,30,31}$ due to its simplicity and capability to obtain enhanced electrical properties. ${ }^{23,29,32,33}$ Subsequently, BCZT ceramic was elaborated by soft chemistry routes like sol-gel, ${ }^{27}$ citrate reaction, ${ }^{34}$ solvothermal, $^{35}$ hydrothermal, ${ }^{36}$ and sol-gel-hydrothermal. ${ }^{37}$ However, relative high temperature is needed to obtain pure and crystalline BCZT powder. We reported the first synthesis of pure and crystalline BCZT near-spherical nanopowder via hydrothermal method at very-low temperature, and the growth mechanism of the BCZT nanoparticles was consisting in the in-situ transformation and/or dissolution-precipitation processes. ${ }^{38}$ It was stated that the electrical properties and the energy storage performances are highly affected by the shape of the ferroelectric material, hence, many works have attempted to elaborate BCZT ceramics with different morphologies namely nanowires and nanofibers. ${ }^{39-43}$ Zhou et al. prepared BCZT nanowires (BCZT-NWs) via two-steps hydrothermal processing and found that the peak power density could be enhanced 9 times by using BCZT-NWs instead of BCZT nanoparticles. ${ }^{44}$ In the present study, BCZT with more complex nanostructures was elaborated through sol-gel followed by twosteps hydrothermal processes. The morphogenesis, the mechanism responsible for the formation of $\mathrm{BCZT}$ multipods alongside with the structural and composition properties are discussed.

\section{Experimental section}

\subsection{Materials}

Titanium isopropoxide $\left(\mathrm{Ti}\left(\mathrm{OC}_{3} \mathrm{H}_{7}\right)_{4}, \geq 98.0 \%\right)$ was obtained from Merk, zirconium n-propoxide solution $\left(\mathrm{Zr}\left(\mathrm{OCH}_{2} \mathrm{CH}_{2} \mathrm{CH}_{3}\right)_{4}, 70\right.$ wt. \% in 1-propanol). Barium acetate $\left(\mathrm{Ba}\left(\mathrm{CH}_{3} \mathrm{COO}\right)_{2}, \mathrm{ACS}\right.$ reagent, 99.0102.0\%) was obtained from Alfa Aesar. Calcium nitrate tetrahydrate ( $\mathrm{Ca}\left(\mathrm{NO}_{3}\right)_{2} \cdot 4 \mathrm{H}_{2} \mathrm{O}, \mathrm{ACS}$ reagent, 99\%), ethanol $\left(\mathrm{CH}_{3} \mathrm{CH}_{2} \mathrm{OH}\right.$, laboratory reagent, 96\%), isopropanol $\left(\left(\mathrm{CH}_{3}\right)_{2} \mathrm{CHOH}, \mathrm{ACS}\right.$ reagent, $\left.\geq 99.5 \%\right)$, sodium hydroxide pellets ( $\mathrm{NaOH}$, puriss. p.a. ACS reagent, $\geq 98 \%$ ), hydrochloric acid $(\mathrm{HCl}, \mathrm{ACS}$ reagent, 37\%) were purchased from Sigma Aldrich. All reagents were used as received without further purification. Deionized water (DI, resistivity of $18.2 \mathrm{M} \Omega \mathrm{cm}^{-1}$ ) was obtained from a PURELAB-classic water purification system (ELGA LabWater) and was used for the experiments conducted in this work.

\subsection{Preparation of ZTO powder}

$\mathrm{Zr}_{0.1} \mathrm{Ti}_{0.9} \mathrm{O}_{2}$ (ZTO) powder were synthesized as reported in ${ }^{38}$ Briefly, appropriate amounts of titanium isopropoxide and zirconium npropoxide were added to isopropanol in $250 \mathrm{~mL}$ round-bottom flask under inert atmosphere $\left(\mathrm{N}_{2}\right)$. After $1 \mathrm{~h}$ of vigorous stirring, deionized water (DI) was added into the solution dropwise to produce a gel designated ZTO. This was washed several times using DI water and ethanol, and dried at $80^{\circ} \mathrm{C}$ for $12 \mathrm{~h}$ to obtain ZTO powder.

\subsection{Preparation of HZTO-NWs}

To produce $\mathrm{H}_{2}\left(\mathrm{Zr}_{0.1} \mathrm{Ti}_{0.9}\right)_{3} \mathrm{O}_{7}$ nanowires (HZTO-NWs), which serves as inorganic template for BCZT preparation, $5 \mathrm{~g}$ of ZTO powder, was dispersed in $100 \mathrm{~mL}$ of $10 \mathrm{M} \mathrm{NaOH}$ aqueous solution in a $150 \mathrm{~mL}$ Teflon-lined stainless-steel autoclave by stirring for $1 \mathrm{~h}$. After sealing and heating at $240{ }^{\circ} \mathrm{C}$ for $48 \mathrm{~h}$, the autoclave was cooled down to room temperature, and the resulting white fluffy suspension was collected by filtration and soaked in $0.2 \mathrm{M} \mathrm{HCl}$ for $10 \mathrm{~h}$ to convert NaZTO to HZTO. The obtained product was centrifuged at 4,000 rpm for $10 \mathrm{~min}$, washed several times with DI water and ethanol, filtered to obtain a fluffy product, then freezedried for $48 \mathrm{~h}$.

\subsection{Preparation of BCZT multipods}

BCZT multipods were prepared through hydrothermal reaction of HZTO-NWs, barium acetate and calcium nitrate tetrahydrate. Under inert atmosphere $\left(\mathrm{N}_{2}\right), 150 \mathrm{mg}$ of HZTO-NWs was dispersed in 35 $\mathrm{mL}$ of barium acetate $(50 \mathrm{mM}$ or $150 \mathrm{mM}$ ) and calcium nitrate tetrahydrate $(8.8 \mathrm{mM}$ or $26.5 \mathrm{mM}$ ) solution and $0.1 \mathrm{M}$ of potassium hydroxide. In both cases, the $\mathrm{Ba}^{2+} / \mathrm{Ca}^{2+}$ ratio is kept to be constant of 2.67 , equals to the stoichiometry ratio of $\mathrm{Ba}$ and $\mathrm{Ca}$ in BCZT. Next, the suspension was sonicated for $10 \mathrm{~min}$ to break the agglomeration. After, the final solution was purged with $\mathrm{N}_{2}$, transferred into a $50 \mathrm{~mL}$-Teflon-lined stainless-steel autoclave, sealed and heated at $180{ }^{\circ} \mathrm{C}$ for different hydrothermal time, starting from $3 \mathrm{~h}$ to $72 \mathrm{~h}$. After the hydrothermal reaction was completed, the sealed autoclave was cooled down to room temperature. The resulting white precipitates were collected by centrifugation at 5,000 rpm for $10 \mathrm{~min}$, soaked in $0.2 \mathrm{M} \mathrm{HCl}$ for $4 \mathrm{~h}$, and washed several times with $0.2 \mathrm{M} \mathrm{HCl}$, DI water and ethanol. 
Then, the final products were dried at $80^{\circ} \mathrm{C}$ for $12 \mathrm{~h}$. The prepared samples were designated as $\mathrm{xByh}$, where $\mathrm{x}$ refers to $\mathrm{Ba}^{2+}$ concentration ( $50 \mathrm{mM}$ or $150 \mathrm{mM}$ ) and y denotes the reaction time in hour.

\subsection{Characterizations}

The room-temperature crystalline structure of the different powders was determined by X-ray diffraction (XRD, Rigaku SmartLab) using a step angle of $0.012^{\circ}$ in the $2 \theta$ range from $5-80^{\circ}$

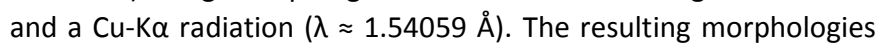
observations in xByh powders were analysed by using a scanning electron microscope equipped with an electron gun (FESEM, JEOL JSM-7600F) and a high-resolution transmission electron microscopy (HRTEM, JEOL - ARM 200F Cold FEG) operating at $200 \mathrm{kV}$ and equipped with a spherical aberration (CS) probe and image correctors with point resolution $0.12 \mathrm{~nm}$. Energy-dispersive X-ray spectroscopy (EDS) elemental mapping was used to probe the compositions of the resulting nanostructures.

\section{Results and Discussions}

\subsection{Morphological transformations}

\subsubsection{Weight-gain of the samples}

The weight gain of the formed oxides as a function of time can provide a macroscopic insight of the transformation kinetics of HZTO-NWs in the presence of barium and calcium species. Fig. 1 gives such evolution for two different conditions of barium/calcium concentration. Both sigmoid curves show a similar behavior, $\Delta \mathrm{m}$ raises rapidly with time, and evolves slowly from $12 \mathrm{~h}$ to $32 \mathrm{~h}$, then become stable from $32 \mathrm{~h}$ to $72 \mathrm{~h}$ of hydrothermal reaction. However, the kinetics is more rapid and the equilibrium weight gain is higher for the highest barium concentration. It is worth noting that the diffusion behavior consists of describing the weight change as a function of square root of diffusion time. Accordingly, in this study, this kind of data presentation was also employed to have an insight on the diffusion of barium and calcium species into HZTONWs.

\subsubsection{Effect of reaction time}

Fig. 2 represents the effect of reaction time on the morphology of xByh samples using $150 \mathrm{mM}$ of $\mathrm{Ba}^{2+}$ and $26.5 \mathrm{mM}$ of $\mathrm{Ca}^{2+}$. HZTONWs with highly anisotropic shape are formed with various widths and lengths, with aspect ratio exceeding 50 as depicted in Fig. 2a and Fig. S1 in the ESI. + After $3 \mathrm{~h}$ of hydrothermal rection, small cubes are grown on the surface of HZTO template (Fig. 2b). A further increasing in reaction time leads to boosted epitaxial growth of cubes on the surface of HZTO wires and then multipods nucleate on the cubes faces until a complete covering of the HZTO template (Fig. 2c). With increasing reaction time, the wire-like shape is progressively lost, while the coarsening of rough wires alongside with morphology degeneration and increasing amounts of loose nanoparticles are observed (Figs. 2d-f).

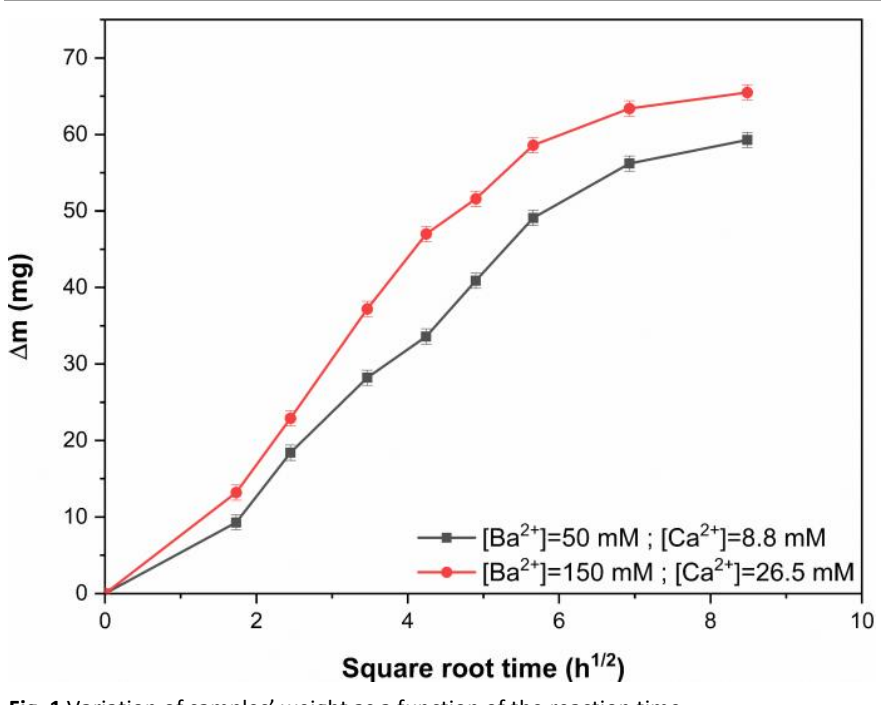

Fig. 1 Variation of samples' weight as a function of the reaction time. 


\section{ARTICLE}

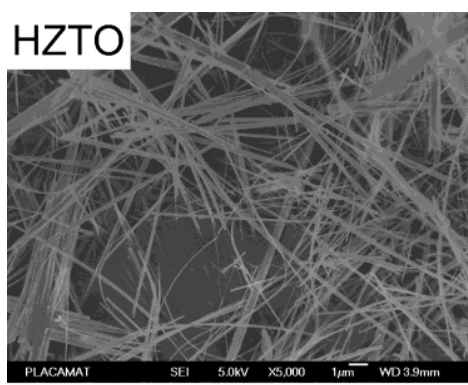

(a)

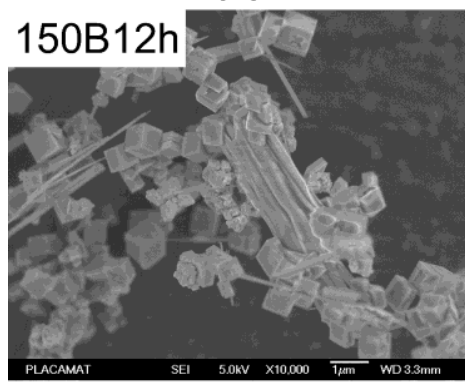

(d)

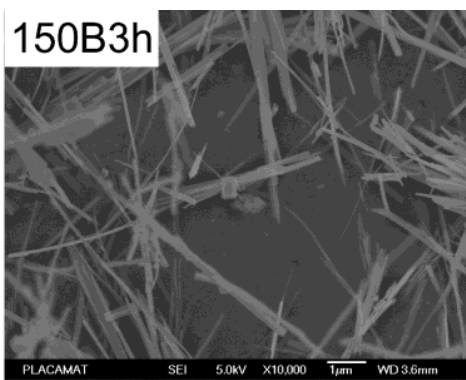

(b)

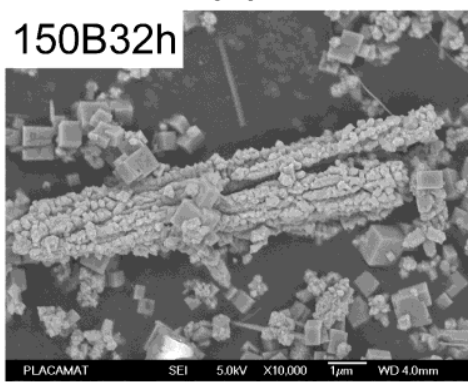

(e)

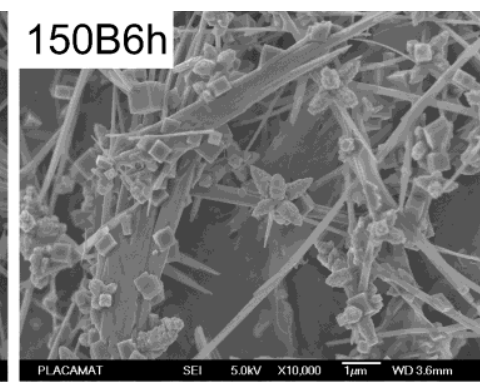

(c)

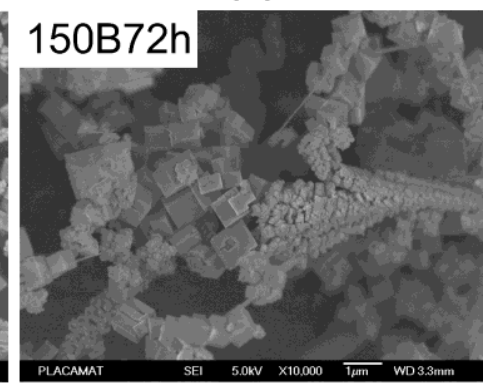

(f)

Fig. 2 FESEM images indicating the effect of reaction time on the morphogenesis of BCZT materials using $150 \mathrm{mM}$ of $\mathrm{Ba}^{2+}$ and $26.5 \mathrm{mM}$ of Ca ${ }^{2+}$. (a) $\mathrm{HZTO}^{2} \mathrm{NWs}$, and after (b) $3 \mathrm{~h}$, (c) $6 \mathrm{~h}$, (d) $12 \mathrm{~h},(\mathrm{e}) 32 \mathrm{~h}$ and (f) $72 \mathrm{~h}$ of hydrothermal reaction.

\subsection{Composition, structure and phases analyses}

Compositional analysis of the resulting morphologies is probed by energy dispersive $\mathrm{X}$-ray spectroscopy (EDS). EDS elemental mapping images could provide the distribution of $\mathrm{Zr}, \mathrm{Ti}, \mathrm{Ca}, \mathrm{Ba}$ and $\mathrm{O}$ elements in the different phases. As observed in Fig. 4, the $\mathrm{Zr}, \mathrm{Ti}$, and $\mathrm{O}$ elements constitute the whole wires in the HZTO-NWs and $\mathrm{Zr}, \mathrm{Ti}, \mathrm{Ca}$, and $\mathrm{O}$ elements constitute the entire cubes, however, in addition to these elements, $\mathrm{Ba}$ is also present in the multipods. Hence, it can be concluded that the wires are HZTO-NWs, the cubes constitute $\mathrm{CaZr}_{\mathrm{y}} \mathrm{Ti}_{1-\mathrm{y}} \mathrm{O}_{3}$ (CZTO), $\mathrm{Ca}_{3}\left(\mathrm{Zr}_{\mathrm{y}} \mathrm{Ti}_{1-\mathrm{y}}\right)_{2} \mathrm{O}_{7}\left(\mathrm{C}_{3}(\mathrm{ZT})_{2} \mathrm{O}_{7}\right)$ and the multipods constitute BCZT phase. It is worth noting that some multipods exhibit small amounts of $\mathrm{Ca}$. More EDS analyses are given in Figs. S2-S4 in the ESI. + The structural properties of each phase

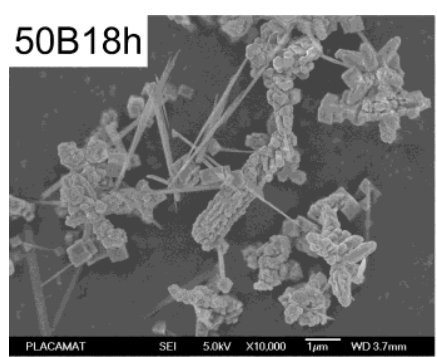

(a)

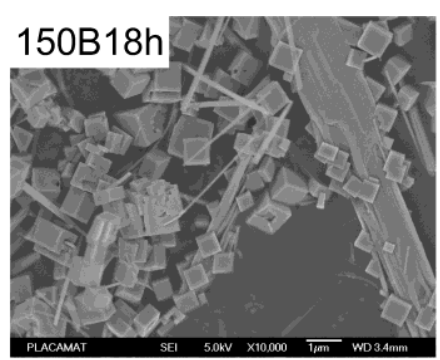

(b)

Fig. 3 FESEM images indicating the effect of A-site concentrations on the morphogenesis of BCZT materials after $18 \mathrm{~h}$ of hydrothermal reaction, using (a) $50 \mathrm{mM}$ of $\mathrm{Ba}^{2+}$ and (b) $150 \mathrm{mM}$ of $\mathrm{Ba}^{2+}$. 


\section{ARTICLE}
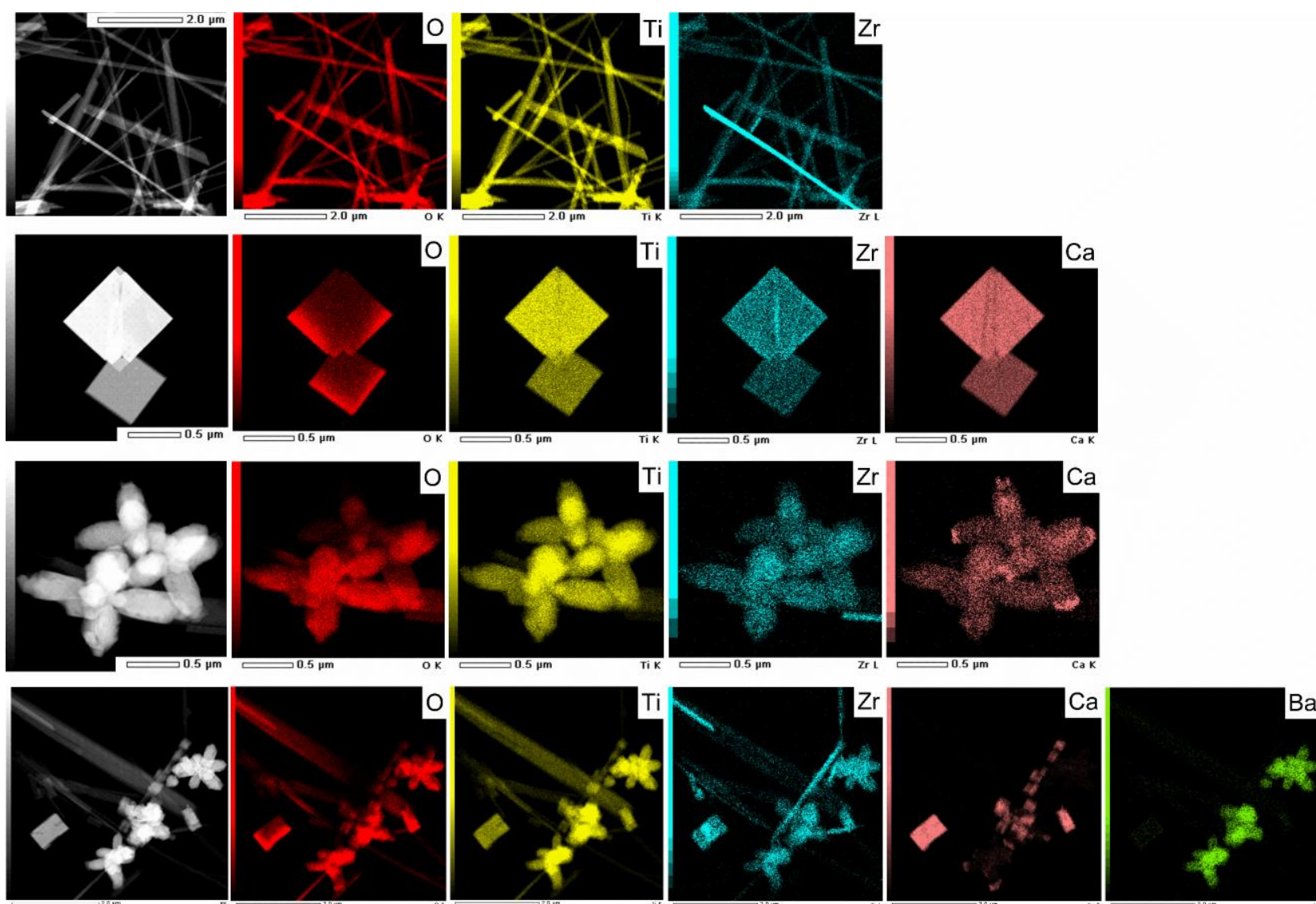

Fig. 4 TEM images and EDS elemental mapping images of wires, cubes and multipods.

The XRD patterns of the powders elaborated using $\left\{\left[\mathrm{Ba}^{2+}\right]=50\right.$ $\left.\mathrm{mM}\left[\mathrm{Ca}^{2+}\right]=8.8 \mathrm{mM}\right\}$ and $\left\{\left[\mathrm{Ba}^{2+}\right]=150 \mathrm{mM},\left[\mathrm{Ca}^{2+}\right]=26.5 \mathrm{mM}\right\}$ at various hydrothermal reaction times are shown in Figs. 5a, b. The XRD patterns can be indexed to monoclinic $(\mathrm{C} 2 / \mathrm{m})$, orthorhombic (Pbnm), orthorhombic $\left(\mathrm{Ccm}_{1}\right)$ and tetragonal (P4mm), for HZTO, CZTO, $\mathrm{Ca}_{3}\left(\mathrm{Zr}_{\mathrm{y}} \mathrm{Ti}_{1-\mathrm{y}}\right)_{2} \mathrm{O}_{7}\left(\mathrm{C}_{3}(\mathrm{ZT})_{2} \mathrm{O}_{7}\right)$ and $\mathrm{BCZT}$, respectively. The XRD pattern of HZTO-NWs is depicted in Fig. S5 in the ESI. + It is observed that whatever the A-site concentrations BCZT desirable phase, unreacted HZTO-NWs, and CZTO and $\mathrm{C}_{3}(\mathrm{ZT})_{2} \mathrm{O}_{7}$ secondary phases are simultaneously present in all samples. Moreover, the secondary phases $\mathrm{CZTO}$ and $\mathrm{C}_{3}(\mathrm{ZT})_{2} \mathrm{O}_{7}$ are remarkably pronounced at high $\mathrm{A}$ site concentration (Fig. $5 \mathrm{~b}$ ). After increasing the reaction time, a reduction of the unreacted HZTO-NWs and the increasing of CZTO and $\mathrm{C}_{3}(\mathrm{ZT})_{2} \mathrm{O}_{7}$ phases were noticed.

The previous observations were quantified to identify the amount of each phase in all samples as shown in Figs. 5c, d. It is found that the desirable BCZT phase with large amount is obtained in samples using low A-site concentration, and especially at reaction time of 6 and $12 \mathrm{~h}$. After increasing the reaction time, the quantity of BCZT phase decreases, and the unreacted HZTO-NWs is reduced. Meanwhile, the secondary phases CZTO and $\mathrm{C}_{3}(\mathrm{ZT})_{2} \mathrm{O}_{7}$ increase. At elevated reaction time $(72 \mathrm{~h}), B C Z T$ phase still present with an amount of $55 \%$. At high A-site concentration, the maximum amount of BCZT is found to be $60 \%$ at $3 \mathrm{~h}$ of reaction. However, after expanding hydrothermal reaction time, a drop of the quantity of BCZT and an increase of the secondary phases CZTO and $\mathrm{C}_{3}(\mathrm{ZT})_{2} \mathrm{O}_{7}$ were noticed (Fig. $5 \mathrm{~d}$ ). Therefore, no single-phase BCZT is obtained through this processing, and the use of low A-site concentration and low reaction time $(\leq 12 \mathrm{~h}$ ) could help to obtain large amount of BCZT phase in the sample. To gain insight into the morphological characteristics of the samples elaborated at prolonged reaction time, Fig. 6 displays FESEM micrographs of 50B72h and $150 \mathrm{~B} 72 \mathrm{~h}$ samples. It is observed that $50 \mathrm{~B} 72 \mathrm{~h}$ powder is composed essentially of multipods, wires, degenerated morphologies and small number of cubes (Fig. 6a), however, 150B72h sample is based on cubes, degenerated morphologies and small number of multipods (Fig. 6b). 


\section{ARTICLE}

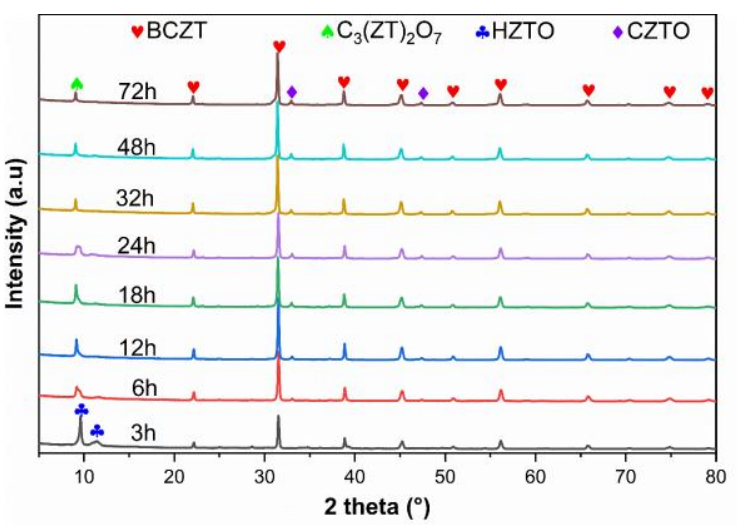

(a)

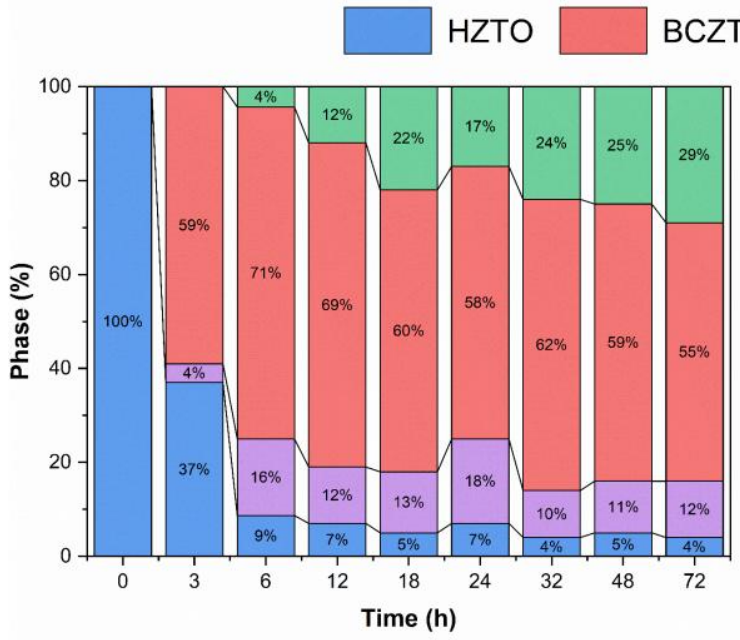

(c)

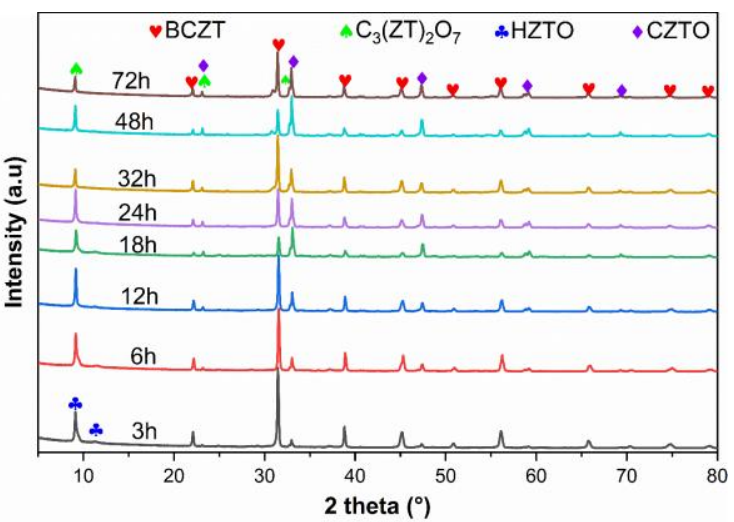

(b)

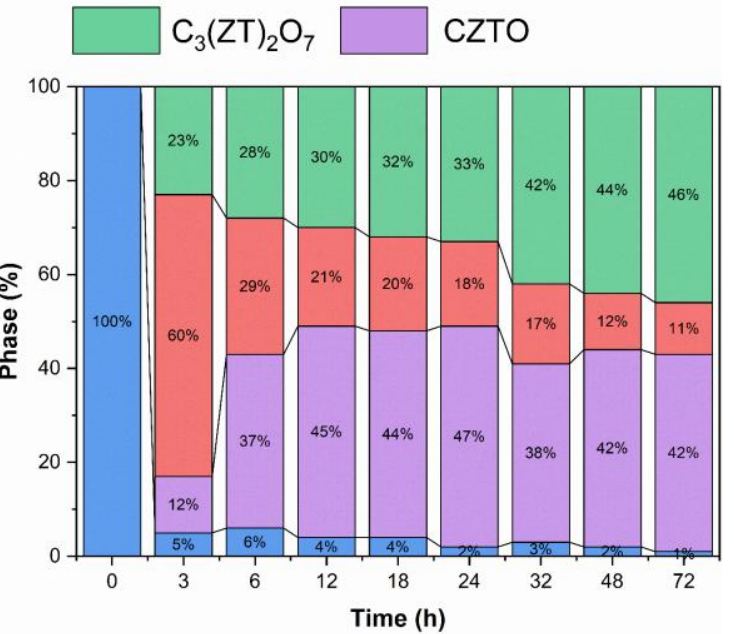

(d)

Fig. $5(\mathrm{a}, \mathrm{b})$ XRD patterns and $(\mathrm{c}, \mathrm{d})$ the composition of different powders elaborated using (a) $\left\{\left[\mathrm{Ba}^{2+}\right]=50 \mathrm{mM},\left[\mathrm{Ca}^{2+}\right]=8.8 \mathrm{mM}\right\}$ and $(\mathrm{b})\left\{\left[\mathrm{Ba}^{2+}\right]=150 \mathrm{mM},\left[\mathrm{Ca}{ }^{2+}\right]=26.5 \mathrm{mM}\right\}$, at various hydrothermal reaction times.

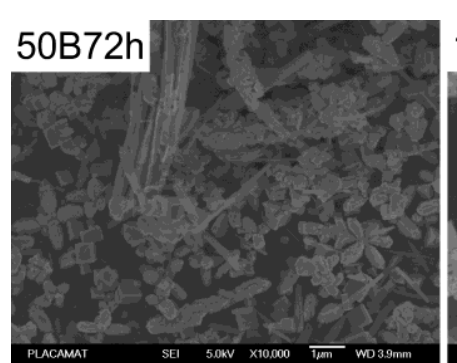

(a)

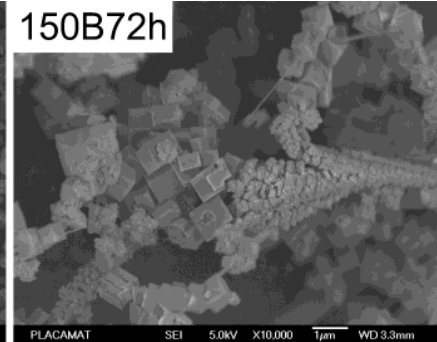

(b)
Fig. 6 FESEM micrographs of 50B72h and 150B72h samples.

It was found that the structure and morphological studies provided important insights into the co-existence of simultaneous phases during the preparation of the samples. Accordingly, to distinguish these phases, SAED diffraction patterns were collected for each morphology. The SAED patterns clearly show that the obtained products possess single-crystalline structures as seen in Fig. 7. According to the SAED patterns, HZTO nanowire, CZTO cube and BCZT multipod are all single-crystals and well crystalline phases. As shown in Fig. 7a, SAED spot distances of the HZTO nanowire are found to be $9.0163 \AA$ and $3.9302 \AA$, which are indexed as (001) and (400) planes, respectively. Likewise, the SAED spot distances of the CZTO cube are calculated to be $0.55 \AA$ and $0.55 \AA$, which are consistent with the (100) and (010) planes, respectively (Fig. 7b). Also, the lattice spacings determined from the diffraction spots of the SAED of BCZT multipod are found to be $3.999 \AA$ and $2.834 \AA$, which match (100) and (011) planes, respectively (Fig. 7c). The corresponding zone axis of each SAED pattern is indicated in the 
top-left corner of each figure. The SAED diffraction patterns are in good agreement with the obtained results of XRD analyses.

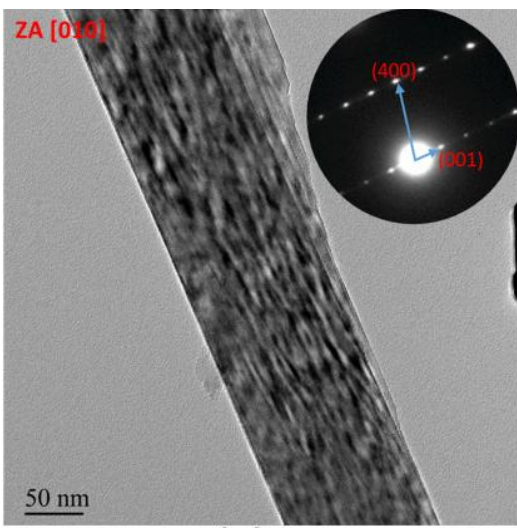

(a)

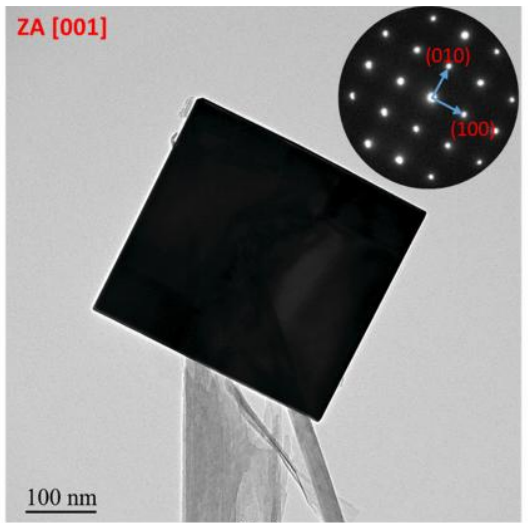

(b)

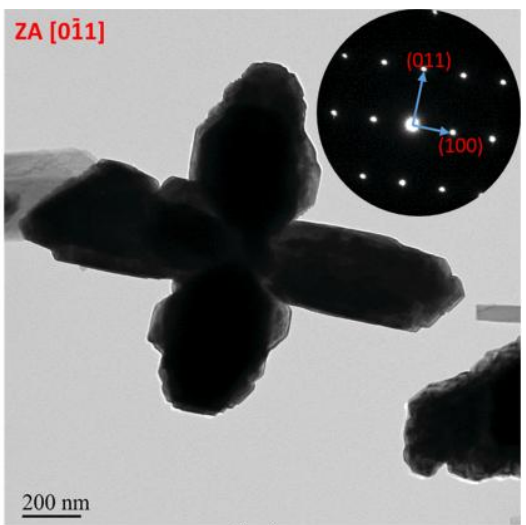

(c)

Fig. 7 TEM images and SAED patterns of (a) HZTO nanowire, (b) CZTO cube, and (c) BCZT multipod.

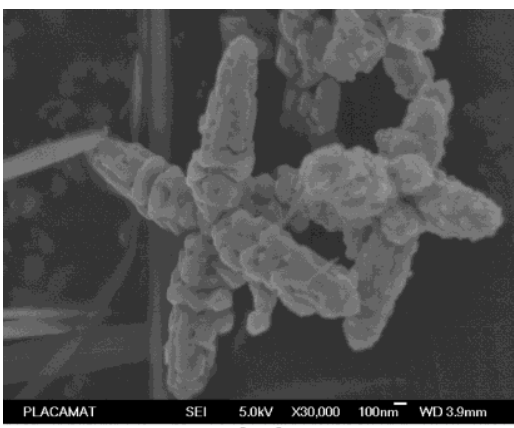

(a)

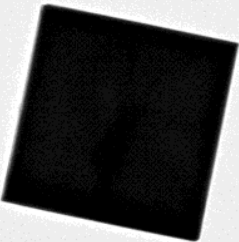

$200 \mathrm{~nm}$

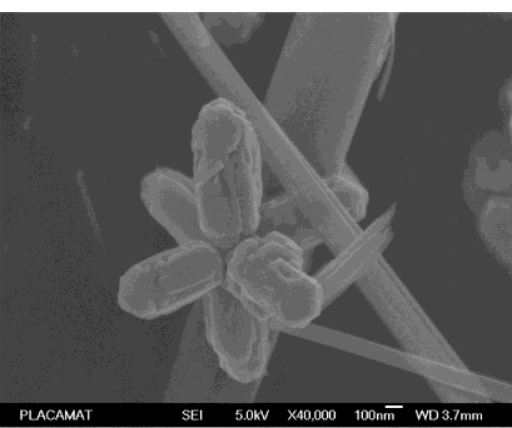

(b)

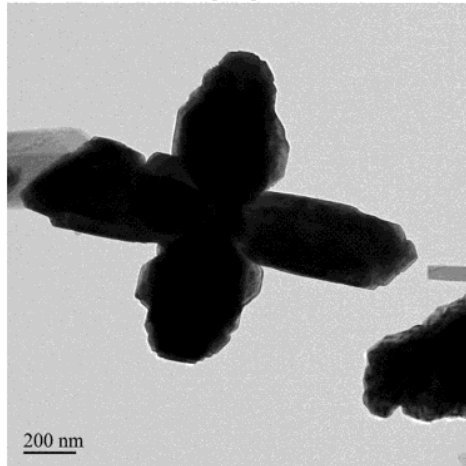

(e)

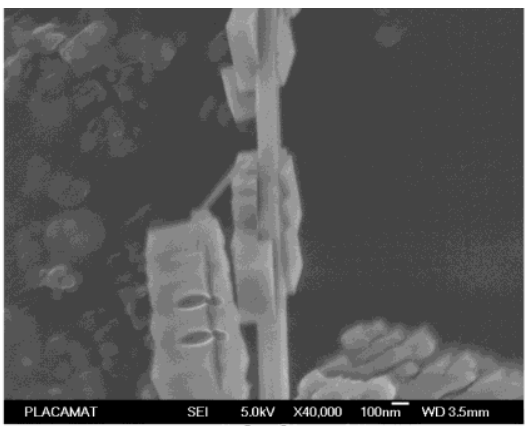

(c)

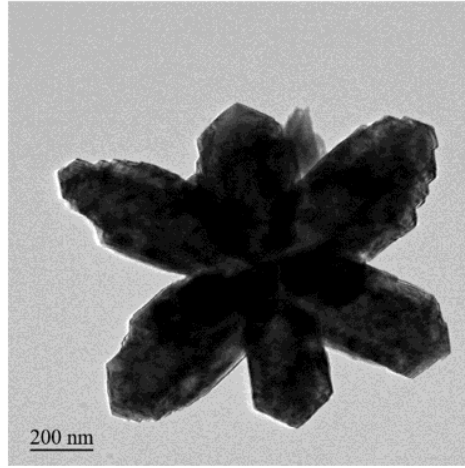

(f)

(d)

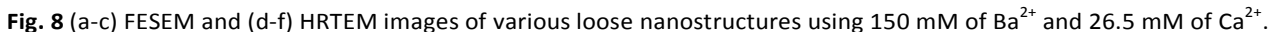

\subsection{Growth mechanism of BCZT multipods}

On the basis of the morphology observations, the domination of the heterogeneous nucleation (cubes and multipods) on the surface of HZTO, leading to the formation of polycrystalline wires with a rough surface, dendritic crystals and some loose nanoparticles (cubes and multipods) (Figs. 8a, d, e, f) indicates that the topochemical transformation of the HZTO template to the BCZT perovskite materials was not happened. ${ }^{11}$ Hence, a dissolution-precipitation processes could be occurred rather than ionic exchange during the hydrothermal reaction. ${ }^{14}$ The most plausible mechanism for the formation of cubes and multipods nanostructures is as follow. First, primary CZTO cubes with good crystallinity, sharp corners and edges and smooth surface (Fig. 8d) decorate the HZTO template via heteroepitaxial growth. These cubes reorient under the influence of the interaction potentials and align along a preferred crystallographic direction, leading to the formation of mesocrystals rather than random aggregates while preserving the initial wire-like morphology of HZTO (Figs. 9a, d). After nucleation, the successive growth of nuclei strongly determines the shape of the nanomaterials, which is thermodynamically driven by the decreasing surface free energy of the generated particles. The nucleation of the cubes on the surface of HZTO-NWs only proceeds at some special sites like as reported by Kalyani et al. ${ }^{45}$ The cubes reorientation step is essential to remove the free energy barrier to 
aggregation. ${ }^{14,46}$ Some of these self-assembly cube arrays, with a size between $200-500 \mathrm{~nm}$, are tightly bound together, and some of them were grown at a certain distance from HZTO surface (Figs. 9a, d). When the diameter of the HZTO template is small enough, two rows of perovskite nanocubes will nucleate on opposite surfaces of the wire as seen in Fig. 8c. More interestingly, two or more nanocubes nuclei are directly fusing together with an edge-to-edge fashion to form larger crystallites, sharing a common crystallographic orientation and uniting at the planar interfaces via a process called oriented attachment (Fig. 10). ${ }^{47,48}$ Generally, for the cubic phase, the relative surface energies $(\gamma)$ are in the order of $V_{\{111\}}<\gamma_{\{100\}}<\gamma_{\{110\}}$ among the lowest index planes. ${ }^{49}$ The presence of inorganics or organics in the solution during the crystal growth process can modify the relative order of surface energies. ${ }^{50}$ The presence of $\mathrm{Ba}^{2+}$ ions could slightly modify the surface energy, and the crystal growth now along $<100\rangle$ direction is much faster than that along the $\langle 111\rangle$ and $\langle 110\rangle$ directions, because the $\{100\}$ facets bore the lowest energy.

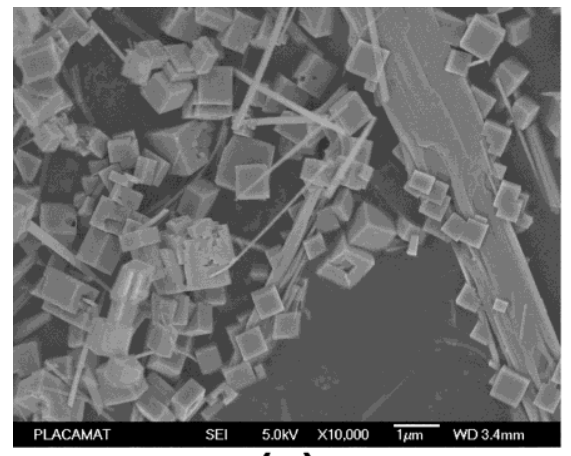

(a)

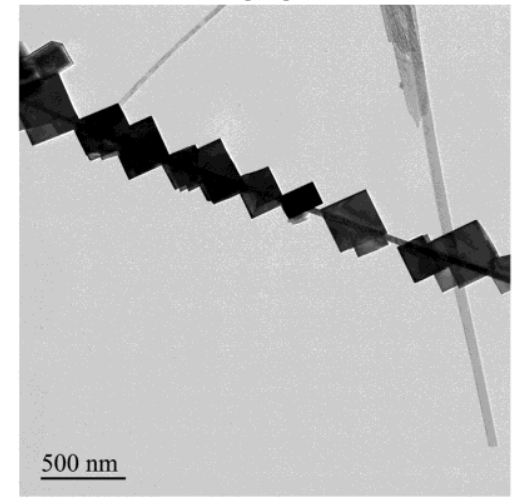

(d)

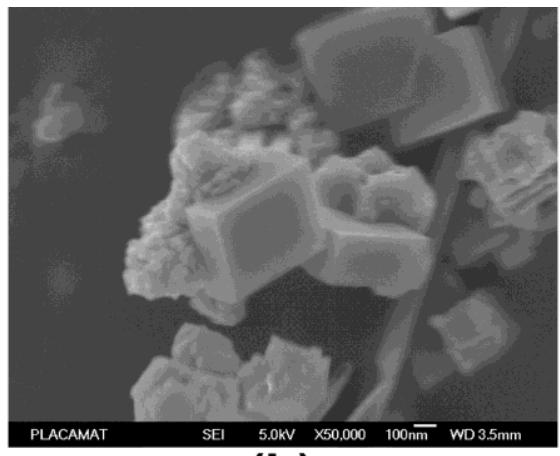

(b)

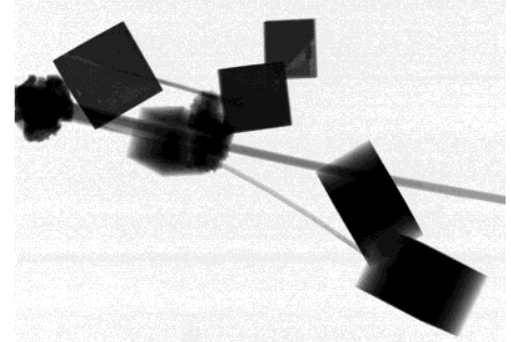

$\underline{0.5 \mu \mathrm{m}}$

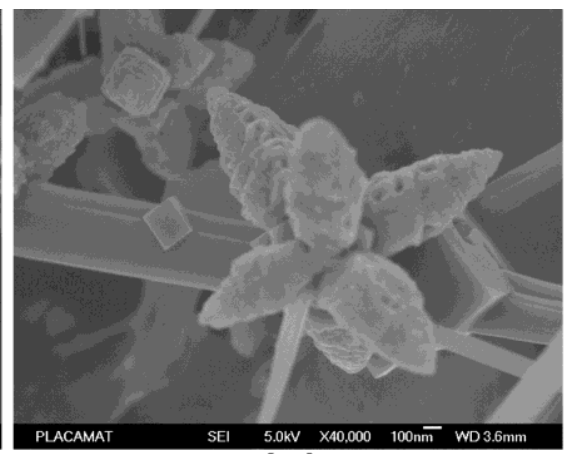

(c)

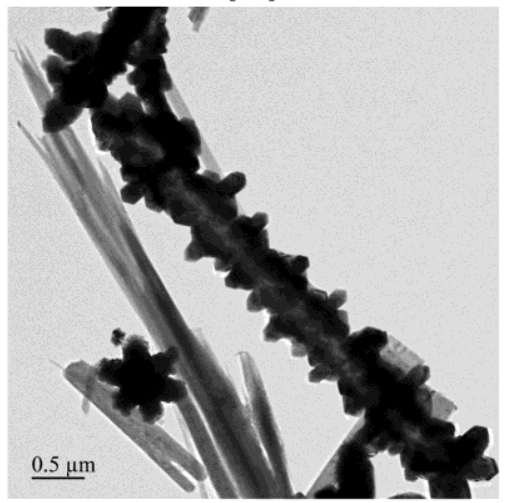

(f)

Fig. 9 (a-c) FESEM and (d-f) HRTEM images indicating the morphogenesis of BCZT materials using $150 \mathrm{mM}$ of $\mathrm{Ba}^{2+}$ and $26.5 \mathrm{mM}^{2}$ of $\mathrm{Ca}^{2+}$.

Yet again, to lower the surface energy, the faces of cubes with $<100>$ directions act as nucleation sites for BCZT multipods (Figs. $9 b$, e) with four to six pods with different growth levels can be observed (Figs. 8a, b, e, f), which grow perpendicular to cubic $\{100\}$ faces while preserving the same orientation (Figs. 9c, f). The octahedral particle is a thermodynamic product with the lowest surface energy and is easily obtained as soon as the octahedral growth habit is controlled and the reaction time is long enough. ${ }^{51}$ The successive morphological evolution depends on the density of surface nuclei. As soon as the HZTO template is totally consumed, a powder composed of loose particles (cubes and multipods) and irregular aggregates is obtained with almost complete loss of the initial wire morphology. The resulted loose particles can be originated from the lattice mismatch or the crystallographic incompatibility between HZTO-NWs and CZTO and BCZT products. ${ }^{45,52}$ A similar evolution was reported in ref. ${ }^{9,14,45}$ These results indicate that BCZT $1 D$ nanostructures cannot be obtained by using HZTO-NWs as templates in hydrothermal process at least under stagnant conditions. It is worthy to note that, despite several attempts to form BCZT nanowires, the topochemical transformation of HZTO-NWs in the presence of barium and calcium species by ion exchange could not be obtained. This suggests that the $\mathrm{Ba} / \mathrm{Ca}$ ions exchange reaction, stated by Zhou et al. as an effective templateassisted synthesis method for the preparation of single phase BCZT nanowires can only occurs in some special experimental conditions and is incompatible with an interface-driven dissolution and crystallization process. ${ }^{44}$ 


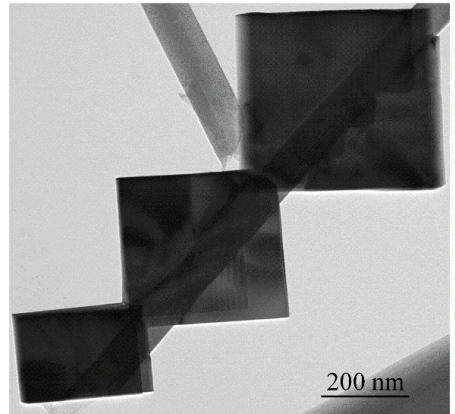

(a)

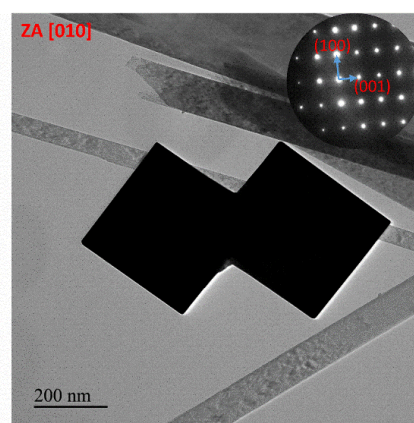

(b)

Fig. 10 HRTEM images of (a) three edge-attached cubes and (b) two edge-attached cubes (SAED pattern in inset).

\section{Conclusions}

Lead-free BCZT nanostructured multipods were designed using sol-gel followed by two-steps hydrothermal processing using HZTO-NWs as inorganic template and calcium nitrate tetrahydrate and barium acetate as A-site sources. The morphogenesis of these nanostructures has been investigated under mild hydrothermal conditions: reaction time of 3-72 h, temperature of $180^{\circ} \mathrm{C}$ and two $\mathrm{A}^{2+}$-concentrations. The growth mechanism of BCZT multipods was investigated, alongside with the composition of all resulting nanostructured phases. BCZT multipods were formed through the epitaxial growth nucleation of CZTO cubes on HZTO-NWs template then perpendicular nucleation of BCZT multipods on the cubes' faces. Besides, it was concluded that the A-site precursor's concentrations played a crucial role in the formation of BCZT multipods, and the desirable BCZT phase was obtained using low A-site concentrations. The reported study is relatively general and can be exploited for the synthesis of other complex perovskite oxides with different nanostructured morphologies.

\section{Conflicts of interest}

There are no conflicts to declare.

\section{Acknowledgements}

The authors gratefully acknowledge the generous financial support of CNRST Priority Program PPR 15/2015 and the European Union Horizon 2020 Research and Innovation actions MSCA-RISE-ENGIMA (No. 778072) and MSCA-RISEMELON (No. 872631). Z. K. acknowledge the Slovenian Research Agency program (P1-0125).

\section{References}

T. R. Shrout and S. J. Zhang, J. Electroceramics, 2007, 19, 111-124.

M. Acosta, N. Novak, V. Rojas, S. Patel, R. Vaish, J. Koruza, G. A. Rossetti and J. Rödel, Appl. Phys. Rev., 2017, 4, 41305. B. Xie, Y. Zhu, M. A. Marwat, S. Zhang, L. Zhang and H. Zhang, J. Mater. Chem. A, 2018, 6, 20356-20364.

M. T. Buscaglia, M. Bassoli, V. Buscaglia and R. Vormberg, J. Am. Ceram. Soc., 2008, 91, 2862-2869.

L. Zhang, X. Wang, H. Liu and X. Yao, J. Am. Ceram. Soc., 2010, 93, 1049-1055.

R. Kavian and A. Saidi, J. Alloys Compd., 2009, 468, 528532.

S. Holliday and A. Stanishevsky, Surf. Coatings Technol., 2004, 188-189, 741-744.

Y. Iriani, M. A. Yasin and R. Suryana, IOP Conf. Ser. Mater. Sci. Eng., 2018, 333, 012047.

Y. Li, X. P. Gao, G. L. Pan, T. Y. Yan and H. Y. Zhu, J. Phys. Chem. C, 2009, 113, 4386-4394.

N. Bao, L. Shen, G. Srinivasan, K. Yanagisawa and A. Gupta, J. Phys. Chem. C, 2008, 112, 8634-8642.

G. Canu and V. Buscaglia, CrystEngComm, 2017, 19, 38673891.

C. Bogicevic, G. Thorner, F. Karolak, P. Haghi-Ashtiani and J. M. Kiat, Nanoscale, 2015, 7, 3594-3603.

Z. Hanani, S. Merselmiz, A. Danine, N. Stein, D. Mezzane, M. Amjoud, M. Lahcini, Y. Gagou, M. Spreitzer, D. Vengust, Z. Kutnjak, M. El Marssi, I. A. Luk'yanchuk and M. Gouné, J. Adv. Ceram., 2020, 9, 210-219.

V. Kalyani, B. S. Vasile, A. lanculescu, A. Testino, A. Carino, M. T. Buscaglia, V. Buscaglia and P. Nanni, Cryst. Growth Des., 2015, 15, 5712-5725.

Z. Wu, S. Yang and W. Wu, Nanoscale, 2016, 8, 1237-1259.

Q. Feng, M. Hirasawa and K. Yanagisawa, Chem. Mater., 2001, 13, 290-296.

M. Zarrabeitia, E. Castillo-Martínez, J. M. López Del Amo, A. Eguía-Barrio, M. A. Muñoz-Márquez, T. Rojo and M. CasasCabanas, J. Power Sources, 2016, 324, 378-387.

G. Wang, X. Huang and P. Jiang, ACS Appl. Mater. Interfaces, 2015, 7, 18017-18027.

M. T. Buscaglia, C. Harnagea, M. Dapiaggi, V. Buscaglia, A. Pignolet and P. Nanni, Chem. Mater., 2009, 21, 5058-5065. Y. F. Zhu, L. Zhang, T. Natsuki, Y. Q. Fu and Q. Q. Ni, ACS Appl. Mater. Interfaces, 2012, 4, 2101-2106.

J. Yang, J. Zhang, C. Liang, M. Wang, P. Zhao, M. Liu, J. Liu and R. Che, ACS Appl. Mater. Interfaces, 2013, 5, 71467151.

D. K. Lee, I. S. Cho, S. W. Lee, D. H. Kim, H. W. Shim, D. W. Kim and K. S. Hong, Eur. J. Inorg. Chem., 2010, 2010, 13431347.

W. Liu and X. Ren, Phys. Rev. Lett., 2009, 103, 257602.

Z. Hanani, D. Mezzane, M. Amjoud, A. G. Razumnaya, S. Fourcade, Y. Gagou, K. Hoummada, M. El Marssi and M. Gouné, J. Mater. Sci. Mater. Electron., 2019, 30, 6430- 
6438.

Z. Hanani, D. Mezzane, M. Amjoud, Y. Gagou, K. Hoummada, C. Perrin, A. G. Razumnaya, Z. Kutnjak, A. Bouzina, M. El Marssi, M. Gouné and B. Rožič, J. Mater. Sci. Mater. Electron., 2020, 31, 10096-10104. M. Maraj, W. Wei, B. Peng and W. Sun, Materials (Basel)., 2019, 12, 3641.

27 J. P. Praveen, T. Karthik, A. R. James, E. Chandrakala, S. Asthana and D. Das, J. Eur. Ceram. Soc., 2015, 35, 17851798.

J. Wu, D. Xiao, W. Wu, Q. Chen, J. Zhu, Z. Yang and J. Wang, J. Eur. Ceram. Soc., 2012, 32, 891-898.

Z. Hanani, S. Merselmiz, D. Mezzane, M. Amjoud, A. Bradeško, B. Rožič, M. Lahcini, M. El Marssi, A. V. Ragulya, I. A. Luk'yanchuk, Z. Kutnjak and M. Gouné, RSC Adv., 2020, 10, 30746-30755. Z. Wang, J. Wang, X. Chao, L. Wei, B. Yang, D. Wang and Z. Yang, J. Mater. Sci. Mater. Electron., 2016, 27, 5047-5058. M. A. Rafiq, M. N. Rafiq and K. Venkata Saravanan, Ceram. Int., 2015, 41, 11436-11444.

S. Patel, D. Sharma, A. Singh and R. Vaish, J. Mater., 2016, 2, 75-86. X. Yan, M. Zheng, X. Gao, M. Zhu and Y. Hou, J. Mater. Chem. C, 2020, 8, 13530-13556. J. Shi, R. Zhu, X. Liu, B. Fang, N. Yuan, J. Ding and H. Luo, Materials (Basel)., 2017, 10, 1093. Z. Hanani, D. Mezzane, M. Amjoud, S. Fourcade, A. G. Razumnaya, I. A. Luk'yanchuk and M. Gouné, Superlattices Microstruct., 2019, 127, 109-117. S. Hunpratub, S. Maensiri and P. Chindaprasirt, Ceram. Int., 2014, 40, 13025-13031. X. Ji, C. Wang, S. Zhang, R. Tu, Q. Shen, J. Shi and L. Zhang, J. Mater. Sci. Mater. Electron., 2019, 30, 12197-12203. Z. Hanani, E. H. Ablouh, M. 'barek Amjoud, D. Mezzane, S. Fourcade and M. Gouné, Ceram. Int., 2018, 44, 1099711000. X. Gao, M. Zheng, X. Yan, J. Fu, M. Zhu and Y. Hou, J. Mater. Chem. C, 2019, 7, 961-967.

40 Z. Pan, L. Yao, J. Zhai, H. Wang and B. Shen, ACS Appl. Mater. Interfaces, 2017, 9, 14337-14346. Q. Chi, G. Liu, C. Zhang, Y. Cui, X. Wang and Q. Lei, Results Phys., 2018, 8, 391-396. C. C. Jin, X. C. Liu, C. H. Liu, Y. Wang, H. L. Hwang and Q. Wang, Mater. Des., 2018, 144, 55-63. K. S. Chary, H. S. Panda and C. D. Prasad, Ind. Eng. Chem. Res., 2017, 56, 10335-10342. Z. Zhou, C. C. Bowland, M. H. Malakooti, H. Tang and H. A. Sodano, Nanoscale, 2016, 8, 5098-5105.

V. Kalyani, B. S. Vasile, A. Ianculescu, M. T. Buscaglia, V. Buscaglia and P. Nanni, Cryst. Growth Des., 2012, 12, 44504456.
R. L. Penn and J. F. Banfield, Geochim. Cosmochim. Acta, 1999, 63, 1549-1557.

C. Dong, Y. Zhou, N. Ta and W. Shen, CrystEngComm, 2020, 22, 3033-3041.

Z. L. Wang, J. Phys. Chem. B, 2000, 104, 1153-1175. X. Lan, J. Zhang, H. Gao and T. Wang, CrystEngComm, 2011, 13, 633-636.

L. Li, L. Tian, Y. Liu, D. Liu, C. Li, M. Liu and C. Wang, CrystEngComm, 2017, 19, 2195-2201.

Z. Zhang, L. Miao, M. Yao, L. Li, F. Zhao, H. Gu, Y. Han, G. A. Sewvandi, Q. Feng, D. Yang, X. Wang and D. Hu, Ceram. Int., 2020, 46, 3675-3687.

$10 \mid$ J. Name., 2012, 00, 1-3 